CTP TAMU-29/00

hep-th/0010116

\title{
Super Yang-Mills Operators from the D3-brane Action in a Curved Background
}

\author{
I.Y. Park, A. Sadrzadeh and T.A. Tran \\ Center for Theoretical Physics, \\ Texas A\&M University, \\ College Station, Texas 77843
}

\begin{abstract}
A consistent truncation of IIB on $S^{5}$ has been obtained in the sector of the metric and the 4-form potential. The ansatz contains 20 scalars and all 15 gauge fields of $\mathcal{N}=8$ gauged supergravity in five dimensions. With this fully non-linear ansatz, the calculations for $n$-point correlators of super Yang-Mills (SYM) theory via AdS/CFT are simpler than those in the literature that use the linear ansatz followed by non-linear field redefinitions. We work out the SYM operators that couple to the scalars by expanding the Dirac-Born-Infeld (DBI) action plus Wess-Zumino (WZ) terms around an $\mathrm{AdS}_{5} \times S^{5}$ background with the metric fluctuations. The resulting operators agree with those based on a superconformal symmetry argument. We discuss the significance of our results.
\end{abstract}




\section{Introduction}

Recently, a consistent fully non-linear reduction of IIB supergravity on $S^{5}$ has been obtained [1, 2] for the $S L(2, R)$-singlet sector comprising the metric and the 4-form potential. In particular, the ansatz contains the scalars $T_{a b}$ in the 20' representation of $S O(6)$ and the 15 gauge fields $A_{(1)}^{a b}$ of $\mathcal{N}=8$ five-dimensional gauged supergravity.

The advantage of having a non-linear ansatz becomes obvious when it comes to computing SYM correlators via AdS/CFT: it is no longer necessary to introduce the non-linear field redefinitions that appeared in the literature as a consequence of using a linear ansatz. Another application of the ansatz we discuss here is to the determination of SYM operators that correspond to given supergravity modes.

In AdS/CFT [3, 4, 5], there are two known ways to determine the SYM operators that correspond to given supergravity modes. In the first approach [5, 6], one considers the representations of the super Lie algebra, $S U(2,2 \mid 4)$, of the fields in SYM theory and IIB supergravity respectively, and matches the supergravity modes with the SYM operators by comparing the various quantum numbers. The other approach, which we will follow in this article, was proposed by Das and Trivedi [7], where they considered the lowest KK mode of the NS-NS 2-form fields polarized along the D3-brane world volume. They worked out the corresponding SYM operators by expanding the DBI action plus WZ terms around an $\mathrm{AdS}_{5} \times S^{5}$ background. They noted that the expansion arund this background, as opposed to a flat background, is crucial in order to obtain the correct SYM operators. A similar method was used in [8, 9]

The relevance of curved backgrounds in AdS/CFT was already noticed in [10, 11] and was further motivated in [12]. It is one of the aims of the present work to consider another supergravity mode, and to work out the SYM operators by following steps that are analogous to those in [7].

The rest of the paper is organized as follows. In section 2, we set the gauge fields

$A_{(1)}^{a b}$ to zero for the purposes of concrete computations, although keeping them does not cause any essential additional complications. The conditions $A_{(1)}^{a b}=0$ then induce fifteen constraints on the scalars $T_{a b}$, since setting the Yang-Mills fields to zero implies that the scalar currents that would excite them must vanish. These constraints can be solved by introducing a diagonal parameterization for $T_{a b}$, with five independent 
diagonal modes. We then compute the action for these diagonal modes. We note that the calculations for the $n$-point correlators are simpler than those in the literature that make use of the linearized ansatz, and which then require non-linear field redefinitions. In section 3, the ansätze for the metric and the 4-form potential are substituted in the Abelian DBI action plus WZ termst. We work out the CFT operators by expanding them to linear order in the diagonal modes. At the end of the section we relax the condition $A_{(1)}^{a b}=0$, and obtain the CFT operators that correspond to the full set of 20 scalars $T_{a b}$. We conclude in section 4 with a discussion of the significance of our results.

\section{Correlators}

Type IIB supergravity can be consistently truncated in $D=10$ such that only the metric and the self-dual 5-form field strength remain, since these are the fields that are singlets under $S L(2, R)$. The non-linear Kaluza-Klein $S^{5}$ reduction ansatz for this sector has been obtained in [1], and is given by

$$
\begin{aligned}
d \hat{s}_{10}^{2}= & \Delta^{1 / 2} d s_{5}^{2}+g^{-2} \Delta^{-1 / 2} T_{a b}^{-1} D \mu^{a} D \mu^{b}, \\
\hat{H}_{(5)}= & \hat{G}_{(5)}+\hat{*} \hat{G}_{(5)}, \\
\hat{G}_{(5)}= & -g U \epsilon_{(5)}+g^{-1}\left(T_{a b}^{-1} * D T_{b c}\right) \wedge\left(\mu^{c} D \mu^{a}\right) \\
& -\frac{1}{2} g^{-2} T_{a c}^{-1} T_{b \ell}^{-1} * F_{(2)}^{a b} \wedge D \mu^{c} \wedge D \mu^{\ell}, \\
\hat{*} \hat{G}_{(5)}= & \frac{1}{5 !} \varepsilon_{a_{1} \cdots a_{6}}\left[g^{-4} U \Delta^{-2} D \mu^{a_{1}} \wedge \cdots \wedge D \mu^{a_{5}} \mu^{a_{6}}\right. \\
& -5 g^{-4} \Delta^{-2} D \mu^{a_{1}} \wedge \cdots \wedge D \mu^{a_{4}} \wedge D T_{a_{5} b} T_{a_{6} c} \mu^{b} \mu^{c} \\
& \left.-10 g^{-3} \Delta^{-1} F_{(2)}^{a_{1} a_{2}} \wedge D \mu^{a_{3}} \wedge D \mu^{a_{4}} \wedge D \mu^{a_{5}} T_{a_{6} b} \mu^{b}\right],
\end{aligned}
$$

where

$$
\begin{aligned}
& U \equiv 2 T_{a b} T_{b c} \mu^{a} \mu^{c}-\Delta T_{a a}, \quad \Delta \equiv T_{a b} \mu^{a} \mu^{b}, \\
& F_{(2)}^{a b}=d A_{(1)}^{a b}+g A_{(1)}^{a c} \wedge A_{(1)}^{c b}, \\
& D T_{a b} \equiv d T_{a b}+g A_{(1)}^{a c} T_{c b}+g A_{(1)}^{b c} T_{a c}, \\
& \mu^{a} \mu^{a}=1, \quad D \mu^{a} \equiv d \mu^{a}+g A_{(1)}^{a b} \mu^{b},
\end{aligned}
$$

\footnotetext{
${ }^{1}$ To the leading order in the momentum expansion, the contributions come only from the DBI action.
} 
and $\epsilon_{(5)}$ is the volume form on the five-dimensional spacetime, whilst $\varepsilon_{a_{1} \cdots a_{6}}$ is the tensor density in six dimensions. $T_{a b}$ is a symmetric unimodular matrix. The ansatz given above is for a general $S^{5}$ reduction, but later we shall choose the spacetime $d s_{5}^{2}$ to be a five-dimensional anti-de Sitter space, $A d S_{5}$.

The resulting Lagrangian and the field equations for $T_{a b}$ and $F_{(2)}^{a b}$ are given in [1]. For simplicity, we consider configurations with $A_{(1)}^{a b}=0$, which in turn enables one to use a diagonal parameterization for $T_{a b}$, as we shall see below. The Lagrangian is

$$
\mathcal{L}=R * \mathbb{1}-\frac{1}{4} T_{a b}^{-1} * d T_{b c} \wedge T_{c e}^{-1} d T_{e a}-\frac{1}{2} g^{2}\left(2 T_{a b} T_{b a}-T_{a a}^{2}\right) * \mathbb{1}
$$

and the field equations are

$$
\begin{aligned}
T_{c[a}^{-1} * d T_{b] c} & =0 \\
d\left(T_{a c}^{-1} * d T_{b a}\right) & =-2 g^{2}\left(2 T_{a c} T_{c b}-T_{a b} T_{c c}\right) \epsilon_{(5)}+\frac{1}{3} g^{2}\left(2 T_{c e} T_{e c}-T_{c c}^{2}\right) \epsilon_{(5)}
\end{aligned}
$$

Since the first equation in (7) imposes fifteen constraints, one can put $T_{a b}$ into the diagonal form

$$
T_{a b}=\operatorname{diag}\left(X_{1}, X_{2}, X_{3}, X_{4}, X_{5}, X_{6}\right) \quad ; \quad \prod_{a=1}^{6} X_{a}=1
$$

We now adopt the parameterization used in [2]

$$
X_{a}=\exp \left(-\frac{1}{2} \vec{b}_{a} \cdot \vec{\varphi}\right)
$$

where $\vec{b}_{a}$ are the weight vectors of the fundamental representation of $S L(6, \mathbf{R})$ and $\vec{\varphi}=\left(\varphi_{1}, \varphi_{2}, \varphi_{3}, \varphi_{4}, \varphi_{5}\right)$ are five independent scalars. The explicit expressions for $\overrightarrow{b_{a}}$ can be taken as follows 2

$$
\begin{aligned}
& \vec{b}_{1}=\left(2, \frac{2}{\sqrt{3}}, \frac{2}{\sqrt{6}}, \frac{2}{\sqrt{10}}, \frac{2}{\sqrt{15}}\right), \quad \vec{b}_{2}=\left(-2, \frac{2}{\sqrt{3}}, \frac{2}{\sqrt{6}}, \frac{2}{\sqrt{10}}, \frac{2}{\sqrt{15}}\right) \\
& \vec{b}_{3}=\left(0,-\frac{4}{\sqrt{3}}, \frac{2}{\sqrt{6}}, \frac{2}{\sqrt{10}}, \frac{2}{\sqrt{15}}\right), \vec{b}_{4}=\left(0,0,-\sqrt{6}, \frac{2}{\sqrt{10}}, \frac{2}{\sqrt{15}}\right) \\
& \vec{b}_{5}=\left(0,0,0,-\frac{8}{\sqrt{10}}, \frac{2}{\sqrt{15}}\right), \quad \vec{b}_{6}=\left(0,0,0,0,-\frac{10}{\sqrt{15}}\right) .
\end{aligned}
$$

\footnotetext{
${ }^{2}$ They also satisfy the following relations: $\vec{b}_{i} \cdot \vec{b}_{j}=8 \delta_{i j}-\frac{4}{3}, \sum_{i=1}^{6} \vec{b}_{i}=0$, and $\sum_{i=1}^{6}\left(\vec{u} . \vec{b}_{i}\right) \vec{b}_{i}=8 \vec{u}$ where $\vec{u}$ is an arbitrary vector.
} 
With the diagonal form of $T_{a b}$, the Lagrangian and equations of motion up to fourth order in $\vec{\varphi}$ are 3

$$
\begin{aligned}
e^{-1} \mathcal{L}= & -\frac{1}{2}(\partial \vec{\varphi}) \cdot(\partial \vec{\varphi})+12 g^{2}+\frac{1}{4} g^{2} \sum_{c=1}^{6}\left(\vec{b}_{c} \cdot \vec{\varphi}\right)^{2}+\frac{1}{24} g^{2} \sum_{c=1}^{6}\left(\vec{b}_{c} \cdot \vec{\varphi}\right)^{3} \\
& -\frac{5}{192} g^{2} \sum_{c=1}^{6}\left(\vec{b}_{c} \cdot \vec{\varphi}\right)^{4}+\frac{1}{128} g^{2}\left[\sum_{c=1}^{6}\left(\vec{b}_{c} \cdot \vec{\varphi}\right)^{2}\right]^{2} \\
= & -\frac{1}{2} \partial_{\mu} \vec{\varphi} \cdot \partial^{\mu} \vec{\varphi}+12 g^{2}+2 g^{2} \vec{\varphi} \cdot \vec{\varphi}+V_{3}+V_{4}, \\
\square \vec{\varphi}= & -4 g^{2} \vec{\varphi}-\frac{1}{8} g^{2} \sum_{a=1}^{6} \vec{b}_{a}\left(\vec{b}_{a} \cdot \vec{\varphi}\right)^{2}+\frac{5}{48} g^{2} \sum_{a=1}^{6} \vec{b}_{a}\left(\vec{b}_{a} \cdot \vec{\varphi}\right)^{3} \\
& -\frac{1}{4} g^{2} \vec{\varphi} \sum_{a=1}^{6}\left(\vec{b}_{a} \cdot \vec{\varphi}\right)^{2}
\end{aligned}
$$

where $V_{3}$ and $V_{4}$ are third-order and fourth-order polynomials in $\vec{\phi}$ respectively. Their explicit forms are presented in the appendix.

In [13], two-point and three-point correlators for various chiral primary operators were computed using a linear ansatz in [14. As a consequence of using the linear ansatz, non-linear field redefinitions were required. The advantage of having a nonlinear ansatz is obvious from ([1]): it renders such field redefinitions unnecessary. One can easily read off two-point and three-point functions using the formulae in [15, 16].

\section{CFT Operators from the Dirac-Born-Infeld Ac- tion}

D-branes are the objects on which open strings can end. They also appear as solitonic solutions of supergravities and string theory. Since these solutions carry mass and charge, one may have to view the open strings as propagating in the curved background produced by the branes to which they are attached [12]. The low-energy effective action for D-branes should then be considered in the same curved background.

The authors of [7] considered the s-wave of the NS-NS $B$-field with non-zero components along the world volume of the D3-brane. They noted that to obtain the correct SYM operators, it is crucial that one expand the DBI action around an $A d S_{5} \times S^{5}$ back-

\footnotetext{
${ }^{3}$ We dropped the Einstein action treating the metric as an $\mathrm{AdS}_{5}$ background.
} 
ground. In this section, we follow [7] and expand the DBI action plus the WZ-terms around an $A d S_{5} \times S^{5}$ vacuum, with the fluctuations parameterized by the diagonal modes $\varphi_{a}$. We then identify the CFT operators, roughly speaking, as the coefficients of $\varphi_{a}$.

Keeping only the terms linear in $\varphi$, the metric ansatz is given by

$$
\begin{aligned}
d s_{10}^{2} \simeq & \left(1-\frac{1}{4} \sum_{i}\left(\mu^{a}\right)^{2} \vec{b}_{a} \cdot \vec{\varphi}\right) d s_{5}^{2} \\
& +\frac{1}{g^{2}}\left(1+\frac{1}{4} \sum_{c} \vec{b}_{c} \cdot \vec{\varphi}\left(\mu^{c}\right)^{2}\right) \sum_{a}\left(1+\frac{1}{2} \vec{b}_{a} \cdot \vec{\varphi}\right)\left(d \mu^{a}\right)^{2} .
\end{aligned}
$$

As previously mentioned, the ansatz quoted in Eq. (11) is for general $S^{5}$ reductions. However, we shall choose the five-dimensional spacetime to be $\mathrm{AdS}_{5}$ for our discussion. The structure of the D3-brane solution of type IIB supergravity in the near-horizon region is such that the "radius" of the $\mathrm{AdS}_{5}$ is the same as that of the internal sphere. Therefore we choose $d s_{5}^{2}$ to be an $\mathrm{AdS}_{5}$ of radius $\frac{1}{g}$. Then we have

$$
d s_{5}^{2}+\frac{1}{g^{2}} \sum_{a}\left(d \mu^{a}\right)^{2}=g^{2} r^{2} \sum_{i}\left(d x^{i}\right)^{2}+\frac{1}{g^{2} r^{2}}\left(d r^{2}+r^{2} \sum_{a}\left(d \mu^{a}\right)^{2}\right) .
$$

The metric ansatz (12) in its written form, i.e. in the $\mu$-coordinate system, does not make manifest the $S O(6)$ covariance of the conformal field theory. A more suitable coordinate system is one that reveals the brane structure more transparently; not surprisingly, such a coordinate system is of Cartesian type,

$$
\mu^{a}=\frac{\Phi^{a}}{r} \quad \text { and } \quad r^{2}=\sum_{a=1}^{6}\left(\Phi^{a}\right)^{2} .
$$

Using the $\Phi$-coordinate system, one can rewrite (12) in the form

$$
d s_{10}^{2}=g^{2} r^{2} f \sum_{i}\left(d x^{i}\right)^{2}+\frac{1}{g^{2} r^{2}} \sum_{a, b=1}^{6} g_{a b} d \Phi^{a} d \Phi^{b}
$$

where

$$
\begin{aligned}
f & \equiv 1-\frac{1}{4 r^{2}} \sum_{a} \vec{b}_{a} \cdot \vec{\varphi}\left(\Phi^{a}\right)^{2} \\
g_{a b} & \equiv \frac{1}{g^{2} r^{2}}\left[\delta_{a b}+\frac{1}{2} \vec{b}_{a} \cdot \vec{\varphi} \delta_{a b}-\frac{1}{r^{2}} \vec{b}_{a} \cdot \vec{\varphi} \Phi^{a} \Phi^{b}+\frac{1}{4 r^{2}} \sum_{c} \vec{b}_{c} \cdot \vec{\varphi}\left(\Phi^{c}\right)^{2} \delta_{a b}\right] .
\end{aligned}
$$

\footnotetext{
${ }^{4}$ It turns out, as we discuss below, that only the DBI action is relevant to the leading order in the derivative expansion.
} 
The action for D3 branes in a general type IIB background was studied in [17, 18, 19, 20. For our discussion, it is enough to keep the metric and the 4-form field since the scalars come only from these. The relevant part of the action is

$$
I=-\int d^{4} \xi \sqrt{-\operatorname{det}\left(G_{i j}+F_{i j}\right)}+\int \hat{C}_{(4)}
$$

where

$$
\begin{aligned}
G_{i j} & =\frac{\partial Z^{M}}{\partial \xi^{i}} \frac{\partial Z^{N}}{\partial \xi^{j}} E_{M}^{A} E_{N}^{B} \eta_{A B}, \\
C_{i j k l} & =C_{M N P Q} \partial_{i} Z^{M} \partial_{j} Z^{N} \partial_{k} Z^{P} \partial_{l} Z^{Q} .
\end{aligned}
$$

It is the 4-form potential $C_{(4)}$ that appears in (17), whereas the ansatz in Eq. (3) and (4) was obtained in terms of the 5 -form field strength, $\hat{H}$. To obtain $C_{(4)}$, consider the metric ansatz in (1). With $A_{(1)}^{a b}=0$, it becomes

$$
d s^{2}=\Delta^{\frac{1}{2}} d s_{5}^{2}+\frac{1}{g^{2}} \Delta^{-\frac{1}{2}} \sum_{a} X_{a}^{-1} d \mu_{a}^{2}
$$

where $\Delta=\sum_{a} e^{-\frac{1}{2} \vec{b}_{a} \cdot \vec{\varphi}} \mu_{a}^{2}$. After some algebra, one can show that the correct $C_{(4)}$ is given by

$$
\begin{aligned}
C_{(4)}= & \frac{1}{2 g}\left(X_{a}^{-1} * d X_{a}\left(\mu^{a}\right)^{2}\right) \\
& -\frac{1}{4 ! g^{4}} \frac{X_{a_{1}} \mu^{a_{1}} \mu^{a_{2}}}{\Delta} \varepsilon_{a_{1} \cdots a_{6}} d \mu^{a_{3}} \wedge \cdots \wedge d \mu^{a_{6}} .
\end{aligned}
$$

Acting with the exterior derivative, the first term gives $\hat{G}_{(5)}$ up to the field equation (7), which is sufficient for our purposes. The second term gives $* \hat{G}_{(5)}$ plus an extra term, which can be cancelled by adding an appropriate term to $C_{(4)}$. However, this term is independent of $\varphi$ and can therefore be dropped in our discussion.

We can in fact also drop the first two terms of equation (20), since we shall eventually consider only the leading order in the derivative expansion. From now on, we may therefore concentrate solely on the contributions coming from the DBI action.

To obtain $G_{i j}$, we substitute (15), (16) and the super vielbeins obtained in [21, 22] into the first equation of (18). For simplicity, we keep only the bosonic fields,

$$
G_{i j}=g^{2} r^{2} f \eta_{i j}+g_{a b} \partial_{i} \Phi^{a} \partial_{j} \Phi^{b}+F_{i j}
$$


After some algebra, one can show that the relevant part of the action is given by

$$
I[\phi] \quad=-\int-\frac{g^{4} r^{2}}{2} \sum_{a} \vec{b}_{a} \cdot \vec{\phi}\left(\Phi^{a}\right)^{2}+\frac{1}{2} \sum_{a b}\left(\frac{1}{2} \vec{b} \cdot \vec{\phi} \delta_{a b}-\frac{1}{r^{2}} \vec{b} \cdot \vec{\phi} \Phi^{a} \Phi^{b}\right) \partial_{i} \Phi^{a} \partial^{i} \Phi^{b}
$$

where the ellipses refer to the terms with higher numbers of derivatives.

The discussion of the full 20 scalars $T_{a b}$, without imposing $A_{(1)}^{a b}=0$, goes very similarly, since eventually one will be interested only in terms that are coupled to $T_{a b}$, but do not have any factors of $A_{(1)}^{a b}$. In fact the computation is almost identical, except that one needs the new parameterization

$$
T_{a b} \equiv\left(e^{S}\right)_{a b}
$$

where $S_{a b}$ is symmetric and traceless. Using this parameterization, one gets

$$
\begin{gathered}
I\left[S_{a b}\right]=-\int g^{4} r^{2} \Phi^{a} \Phi^{b} S_{a b}+\frac{1}{2}\left(-\partial_{i} \Phi^{a} \partial^{i} \Phi^{b}+\frac{1}{r^{2}}\left[\Phi^{a} \Phi^{c} \partial_{i} \Phi^{b} \partial^{i} \Phi^{c}+a \leftrightarrow b\right]\right) S_{a b} \\
-(\text { trace }) .
\end{gathered}
$$

To leading order in the derivative expansion, we have

$$
I\left[S_{a b}\right]=-\int g^{4} r^{2}\left(\Phi^{a} \Phi^{b}-\frac{1}{6} \delta^{a b} \Phi^{c} \Phi_{c}\right) S_{a b}+\cdots
$$

As one can easily show, $S_{a b} \sim \frac{1}{r^{2}}$ in the boundary region, i.e. $r \rightarrow \infty$. Therefore we impose the following boundary condition,

$$
S_{a b} \equiv \frac{1}{r^{2}} S_{a b}^{o}
$$

which leads to the correct CFT operator.

$$
\mathcal{O}^{a b} \equiv\left(\Phi^{a} \Phi^{b}-\frac{1}{6} \delta^{a b} \Phi^{c} \Phi_{c}\right)+\cdots
$$

\section{Conclusion}

In this paper, we considered the 20 of scalars in $\mathcal{N}=8 S O(6)$-gauged supergravity in $D=5$. We showed that using the non-linear Kaluza-Klein ansatz considerably simplifies the calculation of $n$-point CFT correlators in the AdS/CFT correspondence. Then we substituted the ansatz into the Abelian DBI action (plus WZ terms). By expanding 
the action, we could identify the CFT operators that couple to the supergravity modes. The result is given in eq (25) above, and it is in agreement with the CFT operators obtained based on the conformal symmetry argument.

Our work, together with [7], provides evidence for the viewpoint taken in [12]. It might be also viewed as in accordance with the claim made in [24]. These authors argued that the supergravity modes are dual to the "extended" chiral primary operators (CPOs), which contain, in addition to CPOs, their descendents, since the ellipses in (27) will include such quantities.

One may also apply the method of [7] and the present paper to the cases of the M2-brane and M5-brane. Another interesting application will be to the deformations of SYM theory. So far in the literature, one first deforms the $\mathcal{N}=4 \mathrm{SYM}$ theory by adding some operators, and then tries to find the corresponding supergravity solution. However, our results suggest that the procedure may be reversed in those cases where a complete Kaluza-Klein ansatz is known; one may substitute the KK ansatz into the DBI action plus WZ terms, and then work the operators that deform the CFT theory. We hope to report on these issues in the near future.

\section{Acknowledgement}

We thank S. Das, H. Liu, J. Rahmfeld, S. Trivedi and A. Tseytlin for their correspondences. We also thank M. Mihailescu and C. Pope for the useful discussions. 


\section{Appendix : $V_{3}$ and $V_{4}$}

The cubic and quartic terms $V_{3}$ and $V_{4}$ in the scalar potential in equation (11) are given by

$$
\begin{aligned}
V_{3}= & \frac{1}{45} g^{2}\left(30 \sqrt{3} \varphi_{1}^{2} \varphi_{2}-10 \sqrt{3} \varphi_{2}^{3}+15 \sqrt{6} \varphi_{1}^{2} \varphi_{3}+15 \sqrt{6} \varphi_{2}^{2} \varphi_{3}-10 \sqrt{6} \varphi_{3}^{3}\right. \\
& +9 \sqrt{10} \varphi_{1}^{2} \varphi_{4}+9 \sqrt{10} \varphi_{2}^{2} \varphi_{4}+9 \sqrt{10} \varphi_{3}^{2} \varphi_{4}-9 \sqrt{10} \varphi_{4}^{3}+6 \sqrt{15} \varphi_{1}^{2} \varphi_{5} \\
& \left.+6 \sqrt{15} \varphi_{2}^{2} \varphi_{5}+6 \sqrt{15} \varphi_{3}^{2} \varphi_{5}+6 \sqrt{15} \varphi_{4}^{2} \varphi_{5}-8 \sqrt{15} \varphi_{5}^{3}\right) \\
V_{4}= & -\frac{1}{36} g^{2}\left(30 \varphi_{1}^{4}+60 \varphi_{1}^{2} \varphi_{2}^{2}+30 \varphi_{2}^{4}+60 \sqrt{2} \varphi_{1}^{2} \varphi_{2} \varphi_{3}-20 \sqrt{2} \varphi_{2}^{3} \varphi_{3}\right. \\
& +30 \varphi_{1}^{2} \varphi_{3}^{2}+30 \varphi_{2}^{2} \varphi_{3}^{2}+35 \varphi_{3}^{4}+12 \sqrt{30} \varphi_{1}^{2} \varphi_{2} \varphi_{4}-4 \sqrt{30} \varphi_{2}^{3} \varphi_{4} \\
& +12 \sqrt{15} \varphi_{1}^{2} \varphi_{3} \varphi_{4}+12 \sqrt{15} \varphi_{2}^{2} \varphi_{3} \varphi_{4}-8 \sqrt{15} \varphi_{3}^{3} \varphi_{4}+18 \varphi_{1}^{2} \varphi_{4}^{2}+18 \varphi_{2}^{2} \varphi_{4}^{2} \\
& +18 \varphi_{3}^{2} \varphi_{4}^{2}+39 \varphi_{4}^{4}+24 \sqrt{5} \varphi_{1}^{2} \varphi_{2} \varphi_{5}-8 \sqrt{5} \varphi_{2}^{3} \varphi_{5}+12 \sqrt{10} \varphi_{1}^{2} \varphi_{3} \varphi_{5} \\
& +12 \sqrt{10} \varphi_{2}^{2} \varphi_{3} \varphi_{5}-8 \sqrt{10} \varphi_{3}^{3} \varphi_{5}+12 \sqrt{6} \varphi_{1}^{2} \varphi_{4} \varphi_{5}+12 \sqrt{6} \varphi_{2}^{2} \varphi_{4} \varphi_{5}+12 \sqrt{6} \varphi_{3}^{2} \varphi_{4} \varphi_{5} \\
& \left.-12 \sqrt{6} \varphi_{4}^{3} \varphi_{5}+12 \varphi_{1}^{2} \varphi_{5}^{2}+12 \varphi_{2}^{2} \varphi_{5}^{2}+12 \varphi_{3}^{2} \varphi_{5}^{2}+12 \varphi_{4}^{2} \varphi_{5}^{2}+42 \varphi_{5}^{4}\right) \\
& +\frac{1}{2} g^{2}\left(\varphi_{1}^{2}+\varphi_{2}^{2}+\varphi_{3}^{2}+\varphi_{4}^{2}+\varphi_{5}^{2}\right)^{2}
\end{aligned}
$$

\section{References}

[1] M. Cvetič, H. Lü, C.N. Pope, A. Sadrzadeh and T.A. Tran, Nucl. Phys. B586 (2000) 275, hep-th/0003103.

[2] M. Cvetič, S.S. Gubser, H. Lü and C.N. Pope, Phys. Rev. D62 (2000) 086003, hep-th/9909121.

[3] J. Maldacena, Adv. Theor. Math. Phys. 2 (1998) 231, hep-th/9711200

[4] S.S. Gubser, I.R. Klebanov and A.M. Polyakov, Phys. Lett B428 (1998) 105, hep-th/9802109

[5] E. Witten, Adv. Theor. Math. Phys. 2 (1998) 253, hep-th/9802150

[6] S. Ferrara, M.A. Lledo and A. Zaffaroni, Phys. Rev. D58 (1998) 105029, hepth/9805082

[7] S. R. Das and S. P. Trevidi, Phys. Lett. B445 (1998) 142, hep-th/9804149 
[8] S.R. Das and S.D. Mathur, Nucl. Physics. B478 (1996) 561, hep-th/9606185

[9] C.G. Callan, S.S. Gubser, I.R. Klebanov and A.A. Tseytlin, Nucl. Phys. B498 (1997) 65, hep-th/9610172

[10] S. Ferrara, C. Fronsdal and A. Zaffaroni, Nucl. Phys. B532 (1998) 153, hepth/9802203

[11] H. Liu and A.A. Tseytlin, Nucl. Phys. B533 (1998) 88, hep-th/9804083

[12] I.Y. Park, Phys. Lett. B468 (1999) 213, hep-th/9907142

[13] S. Lee, S. Minwalla, M. Rangamani, and N. Seiberg, Adv. Theor. Math. Phys. 2 (1998) 697, hep-th/9806074

[14] H.J. Kim, L.J. Romans and P. van Nieuwenhuizen, Phys. Rev. D32 (1985) 389.

[15] D.Z. Freedman, S.D. Mathur, A. Matusis and L. Rastelli, Nucl. Phys. B546 (1999) 96, hep-th/9804058

[16] W. Mück and K.S. Viswanathan, Phys. Rev. D58 (1998) 041901, hep-th/9804035

[17] M. Cederwall, A. von Gussich, B.E.W. Nilsson and A. Westberg, Nucl. Phys. B490 (1997) 163, hep-th/9610148

[18] E. Bergshoeff and P.K. Townsend, Nucl. Phys. B490 (1997) 145

[19] M. Aganagic, J. Park, C. Popescu and J. Schwarz, Nucl. Phys. B469 (1997) 215, hep-th/9702133

[20] R.R. Metsaev and A.A. Tseytlin, Phys. Lett. B436 (1998) 281, hep-th/9806095

[21] R.R. Metsaev and A.A. Tseytlin, Nucl. Phys. B533 (1998) 109, hep-th/9805028

[22] R. Kallosh and J. Rahmfeld, Phys. Lett. B443 (1998) 143, hep-th/9808038

[23] A.A. Tseytlin, Nucl. Phys. B501 (1997) 41, hep-th/9701125

[24] G. Arutyunov and S. Frolov, Nucl. Phys. B579 (2000) 117, hep-th/9912210 